\section{E-208 RESULTS OF VOLUME-BASED SIZING OF THE WOVEN ENDOBRIDGE (WEB) DEVICE}

K Shah*, T White, I Teron, A Dehdashti, J Katz, H Woo. Neurosurgery, Northwell Health, Manhasset, NY

10.1136/neurintsurg-2020-SNIS.239

Introduction The Woven EndoBridge (WEB) device, an intrasaccular flow-diverter, was recently approved by the FDA to treat wide-neck bifurcation aneurysms. Accurate sizing of the WEB device is of critical importance as it determines procedural safety and successful occlusion. Oversized devices can protrude into the vessel lumen and lead to thromboembolic events, while undersized devices result in inadequate aneurysm occlusion. Conventionally, size selection of the WEB device is based on measurements of the fundus height and neck width on $3 \mathrm{D}$ rotational angiography. However, even multiple, orthogonal linear measurements incompletely describe the three-dimensional morphology of intracranial aneurysms and may mislead size selection of the WEB. An alternative method is volume-based segmentation, minimizing measurement error. The aim of this study was to assess the ability of auto-segmented aneurysm volume to assist in appropriate WEB size selection.

Materials and Methods All patients with an intracranial aneurysm treated with the WEB SL or WEB SLS device between March 2019 and August 2019 were identified for this retrospective study. Aneurysm volumes were determined by using a three-dimensional volume segmentation software on an independent Syngo workstation (Siemens Healthineers AG). Pearson correlation coefficients were calculated for aneurysm auto-segmented volumes and WEB volumes, as well as for aneurysm height $\times$ width and WEB height $\times$ width at the time of initial treatment. Patients underwent follow-up angiography at least 6 months after embolization.

Results Thirty-three aneurysms were evaluated by 3D rotational angiography. By volume, the largest aneurysm was $916.4 \mathrm{~mm}^{3}$, and the smallest was $14.0 \mathrm{~mm}^{3}$, with a median volume of $84.7 \mathrm{~mm}^{3}$. The smallest WEB device used was $4 \times$ 2.6 SLS and the largest WEB device was $11 \times 8$ SL. The correlation with WEB size was larger for auto-segmented aneurysm volumes $(r=0.979)$ compared to height $\times$ width $(r=0.867)$. Using Fisher $r-t o-z$ transformations, we found the difference between the two correlations to be statistically significant $(p=0.0007)$. Out of the patients who have undergone follow-up angiography thus far, 91\% (10/11) have achieved adequate aneurysm occlusion (complete occlusion or residual neck).

Conclusion Aneurysm volumes are highly correlated with WEB volumes, with auto-segmentation volumes displaying statistically significant difference against conventional height by width measurements. These results suggest that the WEB device is more accurately sized using auto-segmented aneurysm volumetric measurements rather than height and width measurements, which may improve long-term occlusion rates.

Disclosures K. Shah: None. T. White: None. I. Teron: None. A. Dehdashti: None. J. Katz: None. H. Woo: None.

\section{E-209 COMPARISON OF ENDOVASCULAR COIL EMBOLIZATION WITH MICROSURGICAL CLIPPING FOR THE TREATMENT OF PARACLINOID ANEURYSMS}

S Chung*, S Chang. Neurosurgery, Eulji University Hospital, Daejeon, Korea, Republic of

10.1136/neurintsurg-2020-SNIS.240

Introduction Paraclinoid aneurysms represent approximately 5\%. Recently, EVT are increasingly used because surgical approach for paraclinoid aneurysms is very hard process. Therefore, although research is rare, we compared clinical outcome for surgical clipping and EVT.

Materials and Methods A retrospective analysis of 91 patients with paraclinoid aneurysm treated with clipping or EVT during 3 years at single center. Outcome was F/U and complications were defined as new neurological deficit, intraoperative rupture, recanalization, or other required re-intervention.

Results 91 paraclinoid aneurysms were treated, including 27 with clipping and 64 with EVT. Most were discovered incidentally but 5 aneurysms were ruptured and isolated visual impairment occurred in 2. About locations, in clipping, ICA dorsal wall and ophthalmic aneurysms were $44 \%$ and in EVT, superior hypophyseal and cavernous $83 \%$. Complete angiographic occlusion was achieved in $87 \%$. Clipping showed in 92.5\%, and EVT in $85.9 \%$. Stent-assisted coiling showed better than coiling alone in occlusion. Good outcomes were $94.5 \%$ in clipping and $98.4 \%$ in EVT. No mortality was found. Complications observed in $25.9 \%$ after clipping, and $7.8 \%$ after EVT (p-value 0.02). Most complications improved during $\mathrm{F} / \mathrm{U}$, however, 1 in EVT and 2 in clipping were left as permanent sequalae (p-value 0.003). EVT, including coiling alone, stent-assisted coiling showed fewer intracranial hemorrhage complications, fewer NCs, and a lower unfavorable visual outcomes rate benefits over than surgical clipping. No significant difference showed in characteristics, clinical

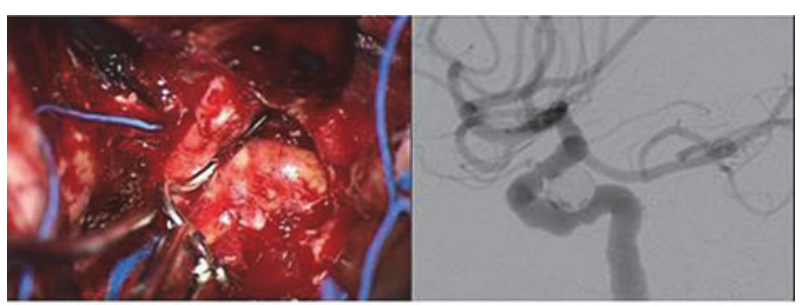

Aneurysm locations
Locations in clipping

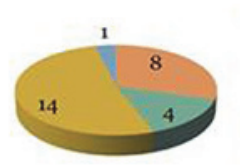

$1+2-44.4 \%$

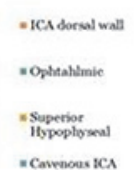

wCavenoas tCa

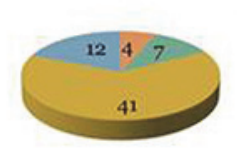

$3+4-82.8 \%$
Abstract E-209 Figure 1 
outcome, occlusion rate and reoperation. Moreover, clipping and stent-assisted coiling all showed higher occlusion rates compared with coiling alone.

Conclusion Definitely, EVT is the mainstream treatment for paraclinoid aneurysms. Surgical clipping showed a higher complication risk than EVT. Clipping might be considered to be an alternative option for paraclinoid aneurysms if EVT are for hard hurdle because many complications were temporary, and occlusion tendency of clipping was also good.

Disclosures S. Chung: None. S. Chang: None.

\section{E-210 AN IN VITRO STUDY OF INTRANEURYSMAL PRESSURE INCREASES DURING CONTRAST INJECTIONS IN CEREBRAL ANGIOGRAPHY}

${ }^{1} S$ Marfoglio, ${ }^{1}$ B Kovarovic, ${ }^{2}$ W Hou, ${ }^{3} \mathrm{D}$ Fiorella, ${ }^{3} \mathrm{C}$ Sadasivan*. ${ }^{*}$ Biomedical Engineering, Stony Brook University, Stony Brook, NY; ${ }^{2}$ Family, Population and Preventive Medicine, Stony Brook University, Stony Brook, NY; ${ }^{3}$ Neurological Surgery, Stony Brook University, Stony Brook, NY

\subsection{6/neurintsurg-2020-SNIS.241}

Introduction During angiography, the contrast bolus injected into a vessel can cause substantial changes in baseline pressures and flows. ${ }^{1}$ There have been sparse case reports about aneurysmal re-bleeds during angiography. ${ }^{2}$ A physician survey of injection rates used during angiography showed that $81 \%$ of respondents considered the impact of injection rate on aneurysm rupture to be negligible. ${ }^{3}$ The goals of this study were to record intraneurysmal pressure changes during contrast injections and to evaluate the effect of injection conditions on intraneurysmal pressure changes.

Methods A silicone replica of a complete circle of Willis model with ophthalmic, anterior communicating, and basilar tip aneurysms was connected to a pulsatile flow pump (Vascular Simulations, Stony Brook, NY, figure 1). Catheters were placed in either the right internal carotid or right vertebral artery and a total of 144 injections were performed by varying 4 different parameters: Catheter Size, Injection Rate, Injection Time, mean Baseline Blood Flow rate (table). The effect of each of the four injection parameters on percentage increase in aneurysm pressures was statistically assessed using ANCOVA; baseline pressure was considered a covariate.

Results The mean intraneurysmal pressure during injection $(84.5 \pm 10.8 \mathrm{mmHg})$ was significantly higher than the mean pressure before injection $(80.4 \pm 10.6 \mathrm{mmHg})(\mathrm{p}<0.0001)$. The percentage increase in mean and systolic aneurysm pressures was $5.1 \pm 3.6 \%$ and $3.4 \pm 2.6 \%$, respectively. The percentage increase in aneurysm pressure was significantly greater $(p<0.0001)$ at high Injection Rates versus low Injection Rates $(6.6 \pm 4 \%$ versus $3.7 \pm 3 \%)$. Increase in aneurysmal pressure was also significantly affected by Catheter Size in most of the statistical comparisons $(p<0.0001)$. Injection Time $(p>0.2)$ and Baseline Blood Flow rate $(p>0.3)$ had no significant effect on the increase in intraneurysmal pressure.
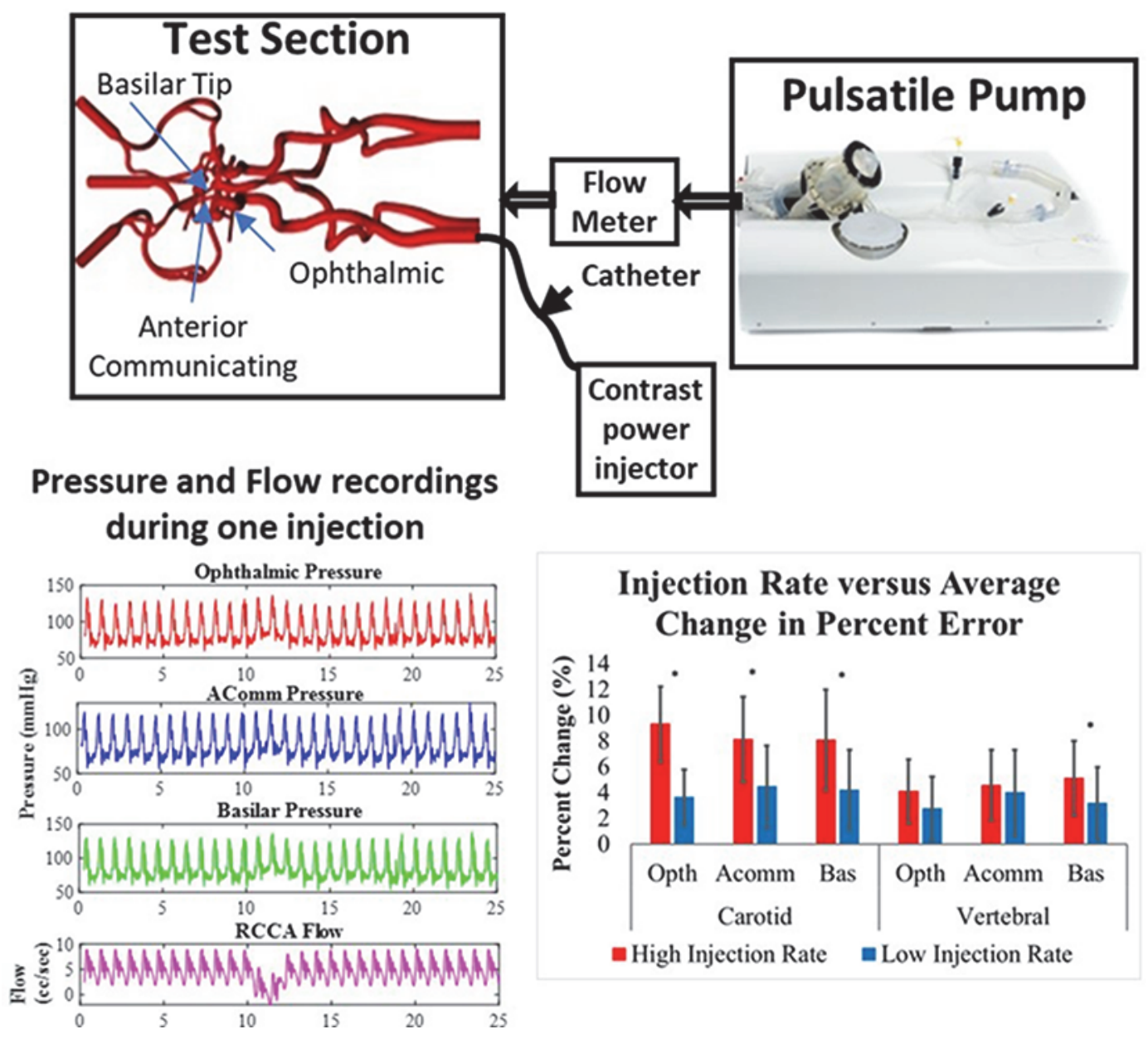

Injection Rate versus Average Change in Percent Error

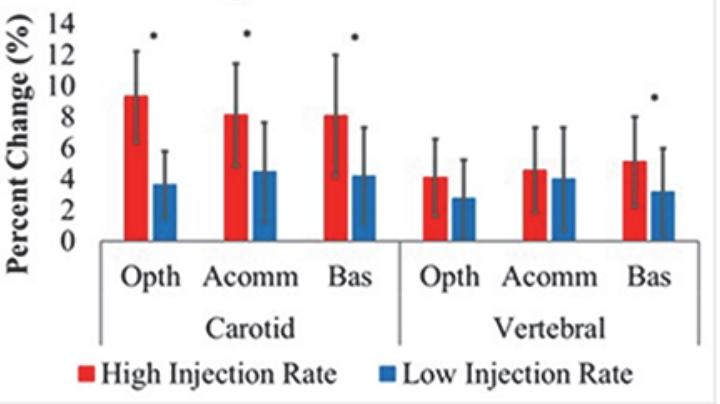

Abstract E-210 Figure 1 